\title{
ATENDIMENTO PSICOLÓGICO E DEMANDAS DISCENTES A PARTIR DE SERVIÇOS EM FACULDADES PRIVADAS DO NORTE DO PAÍS
}

\author{
ATENCIÓN PSICOLÓGICA Y DEMANDAS DE LOS ESTUDIANTES EN \\ SERVICIOS DE UNIVERSIDADES PRIVADAS DEL NORTE DEL PAÍS
PSYCHOLOGICAL CARE AND DISCIPLINARY DEMANDS FROM SERVICES IN PRIVATE FACULTIES IN THE NORTH OF THE COUNTRY

\author{
Núbia Roberta AYRES ${ }^{1}$ \\ Cleber LIZARDO DE ASSIS ${ }^{2}$
}

RESUMO: Se por um lado, os discentes que frequentam o ensino superior no país, apresentam diversas demandas, especialmente psicossociais, as faculdades procuram oferecer serviços de atendimento psicológico como parte do dispositivo institucional. Este artigo tem por objetivo discutir as possibilidades de atuação do psicólogo junto a estudantes da Educação Superior a partir de uma tipologia de casos nesses serviços. Metodologicamente, se utiliza de uma pesquisa bibliográfica dificuldade de aprendizagem dos acadêmicos do nível superior, as queixas do fracasso no âmbito do processo de escolarização e os fatores associados, além de conceitos de psicoterapia breve instrumentalizada a este tipo de atendimento. Adota-se ainda como vinhetas, elementos coletados de documentos de atendimentos psicológicos em 02 instituições de ensino superior. Pode-se perceber que se faz necessário na diminuição das tensões e problemas presentes na Educação Superior por meio dos acadêmicos à inserção de uma perspectiva psicológica, aliada a práticas pedagógicas, uma vez que foi observado que a dificuldade de aprendizagem dos acadêmicos estudados estava diretamente relacionadas a fatores intrínsecos, ou seja, emocionais.

PALAVRAS-CHAVE: Serviço de atendimento psicológico. Ensino superior. Psicoterapia breve. Dificuldade de aprendizagem.

RESUMEN: Por un lado, los estudiantes que asisten a la educación superior en el país, tienen diferentes demandas, especialmente psicosocial, colegios buscan brindar servicios psicológicos como parte del dispositivo institucional. Este artículo tiene como objetivo discutir las posibilidades de psicólogo con los estudiantes de educación superior a partir de una tipología de los casos estos servicios. Metodológicamente, utilizando una dificultad literatura de la formación académica de nivel superior, las

\footnotetext{
${ }^{1}$ Docente do curso de Psicologia da FACIMED-RO. Especialista em Avaliação Psicológica pelo IPOG. E-mail: nubiaayres_cacoal@hotmail.com.

${ }^{2}$ Faculdade Jesuíta de Filosofia e Teologia (FAJE) - Minas Gerais. Pós-doutorando em Filosofia pela FAJE-MG. Pesquisador integrante do Grupo de Pesquisa Práticas de Saúde em Clínica Ampliada na Contemporaneidade-PUCMG. E-mail: kebelassis@yahoo.com.br
} 
quejas de fallo en el proceso de la educación y los factores asociados, y los conceptos de psicoterapia breve instrumentalizados para este tipo de atención. Se adoptó como todavía viñetas recogen elementos de tratamiento psicológico de los documentos en 02 instituciones de educación superior. Se puede observar que es necesario disminuir las tensiones y los problemas actuales en la educación superior a través de los académicos para la inserción de un punto de vista psicológico, combinado con las prácticas de enseñanza, ya que se observó que la dificultad de aprender los estudiosos estudiados estaban directamente relacionados factores intrínsecos o emocional.

PALABRAS-CLAVE: Servicio psicológico. Enseñanza superior. Psicoterapia breve. Problemas de aprendizaje.

ABSTRACT: If, on the one hand, students attending higher education in the country present several demands, especially psychosocial, faculties seek to offer psychological services as part of the institutional arrangements. The purpose of this article is to discuss the psychologist 's possibilities with Higher Education students based on a typology of cases in these services. Methodologically, we used a bibliographical research difficulty of learning of the higher level academics, the complaints of failure in the scope of the schooling process and the associated factors, as well as concepts of brief psychotherapy instrumentalized to this type of care. It is also adopted as vignettes, elements collected from documents of psychological care in 02 institutions of higher education. It can be seen that it is necessary to reduce the tensions and problems present in Higher Education through the academic to the insertion of a psychological perspective, allied to pedagogical practices, once it was observed that the learning difficulties of the studied students were directly related To intrinsic, that is, emotional factors.

KEYWORDS: Psychological attention service. Higher education. Brief psychotherapy. Learning difficulty.

\section{Introdução}

A demanda pela qualificação profissional tem refletido na busca constante pelo Ensino Superior, as últimas décadas tem ilustrado o crescente ingresso dos acadêmicos. Contudo, junto com ao crescente ingresso e demanda de acadêmicos vem acompanhado de situações até então inéditas como a necessidade do acompanhamento psicológico.

As instituições de Ensino Superior (IES) voltam seu olhar para o cuidado e atenção a qualidade de vida do acadêmico de modo a preservar e atender as demandas específicas da saúde mental, realizando atendimentos psicoterápicos breves. Haja vista que as situações emocionais entre outros fatores intrapsíquicos têm interferido diretamente no rendimento deste aluno. 
Desse modo, esse artigo tem por objetivo discutir as possibilidades de atuação do psicólogo junto a estudantes da Educação Superior a partir de uma tipologia de casos nesses serviços. Utilizando-se da pesquisa bibliográfica esse texto versa sobre a discussão dos aspectos relacionados teoricamente sobre a Psicoterapia breve debruçando-se sobre os estudos tocantes aos trabalhos publicados no campo da aprendizagem de forma a discutir sobre as contribuições as dificuldades de aprendizagem presentes no Ensino Superior. Sustentando assim a compreensão das queixas de aprendizagem manifestadas nos atendimentos realizados nas instituições privadas e discutidas nesse texto.

\section{Psicoterapia breve}

Abordar a origem das psicoterapias breves não é tarefa das mais fáceis, uma vez que estas são apresentadas como modelos de expansão de técnicas diversas e, até mesmo, com base teórica e conceitual variada. De modo geral, as psicoterapias breves caracterizam-se por ampliar a capacidade operativa do terapeuta, oferecendo técnicas variadas e específicas a cada caso (KNOBEL, 1986). Já Fiorini (2008, p. 18), considerando a novidade que representam algumas técnicas dentro da psicoterapia breve, salienta que:

[...] essas psicoterapias estão passando pelo teste da experiência (que já não é tão nova), apresentando resultados que permitem construir algumas hipóteses provisórias. Portanto, parece oportuno considerar ao menos a possibilidade de que essas práticas transcendam o quadro de um empirismo obscuro, encontrem uma racionalidade que as legitime, as torne coerentes $\mathrm{e}$ as constitua em técnicas individualizáveis, dotadas de sentido, indicadas cientificamente, e não meros recursos acidentais para uma emergência socioeconômica e sanitária.

O autor enfoca o fato de as psicoterapias breves já estarem sendo praticadas há algum tempo e, no entanto, ainda carecerem de resultados e sistematização que as legitimem. Já foi dito que o cenário atual demanda novas técnicas, ainda assim, não podem ser as demandas diferenciadas o palco de apenas tentativas, o cenário deve servir de base para a criação e legitimação de técnicas mais apropriadas para a atualidade.

Knobel (1986) salienta que muitos pacientes não podem se beneficiar da técnica psicanalítica ou não podem chegar a obtê-la, assim, a psicoterapia breve passa a atuar 
por uma imposição destes pacientes em face de uma necessidade real e cada vez mais há profissionais aplicando esta técnica. Já Hegenberg (2010) solicita parcimônia na aplicação da Psicoterapia Breve, salientando que só poderá ser utilizada se o paciente for capaz de focalizar, de reconhecer os limites de tempo e de poder aproveitar a terapia neste menor espaço temporal.

No que se refere ao profissional, à figura do terapeuta, Fiorini (2008, p. 28) salienta que:

Um terapeuta que introduz na sessão suas iniciativas para a confrontação, que promove o diálogo, é um dos componentes originais dessa técnica, talvez o mais específico para sua caracterização. Sem essa intervenção abertamente participante do terapeuta, o curso de uma terapia breve, entregue à espontaneidade do paciente, passa por sérias dificuldades. A falta de uma compreensão adequada desse fato fundamental por parte do terapeuta parece ser detectada com frequência como um dos determinantes fundamentais de fracassos em tratamentos breves.

\section{Aprendizagem}

Aprendizagem é um artifício de alteração do comportamento obtido por intermédio da experiência edificada por variáveis emocionais, neurológicos, relacionais e ambientais. Aprender é a consequência da influência mútua entre composições mentais e o meio ambiente. De acordo com a nova solenidade educacional, situada na aprendizagem, o docente é coautor do procedimento da aprendizagem dos alunos. Nesse aspecto centrado na aprendizagem, a informação é arquitetada e reconstruída continuamente (JOSÉ; COELHO, 1989). Já para Borges (2008), a aprendizagem é um conjunto de regras e conhecimento com função de nortear uma atividade. A aprendizagem é descrita como aquisição de conhecimento promovendo mudança de comportamento:

Aprendizagem é o processo pelo qual uma atividade tem origem ou é modificada pela reação a uma situação encontrada, desde que as características da mudança de atividade não possam ser explicadas por tendências inatas de respostas, maturação ou estados temporários do organismo (por exemplo, fadiga, drogas, etc.) (HILGARD, 1973, p.3).

Entretanto, para que ocorra a aprendizagem, é necessária uma série de estruturas cerebrais, devidamente maturadas. Segundo Drouet (2001), sete fatores são fundamentais para que ocorra a aprendizagem, sendo: saúde física, saúde mental, 
motivação, prévio domínio, maturação, inteligência, concentração e memória. Em linhas gerais, entende-se a saúde física como questões físicas do organismo, todo o processo de desenvolvimento do indivíduo. A saúde mental está relacionada às condições mentais do indivíduo, se o mesmo apresenta uma qualidade de vida emocional boa, ausência de qualquer doença mental.

A atenção ou concentração são as formas que o indivíduo focaliza uma determinada atividade, o tempo e qualidade do foco na ação, manter-se envolvido com a atividade ou conteúdo a ser aprendido. E, por fim, mas não menos importante, a memória, função de armazenamento da informação, comparação do conteúdo gravado anteriormente e adquirido com a nova aquisição. Todo o processo de desenvolvimento humano contribui para a aprendizagem, ao passo que é caracterizado por três aspectos fundamentais (RIBEIRO, 2003; PAPALIA, 2000).

A questão cognitiva, física e social são dimensões do desenvolvimento humano inter-relacionadas, ou seja, uma influência a outra em seu desenvolvimento. Tais dimensões são de suma importância para a aprendizagem, o desenvolvimento físico é importante desde a primeira infância até sua maturação do indivíduo. (PAPALIA, 2000).

Alguns autores como Ciasca (1999), Fonseca (2009), Zonolla (2012), diferenciam a dificuldade de aprendizagem por questões físicas, neurológicas, emocionais, cognitivas, educacionais e sociofamiliar. No quadro abaixo, apresentamos os fatores que possivelmente contribuem para as dificuldades de aprendizagem dos indivíduos:

Quadro 1: Fatores que contribuem para a dificuldade de aprendizagem.

\begin{tabular}{ll}
\hline \multicolumn{1}{c}{ Fatores } & \multicolumn{1}{c}{ Descrição } \\
\hline Físicas e Sensoriais & $\begin{array}{l}\text { Perturbações ou distúrbios transitórios, } \\
\text { perturbações ou distúrbios permanentes, causas } \\
\text { sensoriais ou perceptivas; }\end{array}$ \\
Causas Neurológicas & $\begin{array}{l}\text { Disgrafia, disortografia, discalculia e dislexia. } \\
\text { Distúrbios da motricidade: Hiperatividade); }\end{array}$ \\
Causa Emocional & $\begin{array}{l}\text { Angústia e depressão, desvios de comportamento: } \\
\text { comportamento agressivo e comportamento } \\
\text { retraído, medos e fobias; }\end{array}$ \\
Causas Intelectuais e Cognitivas & $\begin{array}{l}\text { Desenvolvimento da inteligência e cognição "os } \\
\text { superdotados, os subdotados ou infradotados"; }\end{array}$ \\
Causa Educacionais & $\begin{array}{l}\text { Dispedagogia (Influência do meio escolar no } \\
\text { aparecimento das Dificuldades de Aprendizagem); } \\
\text { Ausência de recursos financeiros e influência } \\
\text { negativa de familiares, professores e colegas. }\end{array}$ \\
\hline
\end{tabular}

Fonte: CIASCA (1999); FONSECA (2009); ZONOLLA (2012) - adaptado pelos autores. 


\section{Dificuldade de aprendizagem no Ensino Superior}

Atualmente as discussões e os parâmetros de avaliação da Educação Superior sinalizam para a concepção de uma educação profissional de excelência na qual são exigidas competências e habilidades necessárias aos egressos do Ensino Superior nas diversas áreas do conhecimento, por meio das Diretrizes Curriculares Nacionais dos cursos de graduação. Em contrapartida à busca de uma educação que atenda aos anseios da sociedade do conhecimento e da informação e dos padrões de qualidade definidos pelo Ministério da Educação, a problemática do desenvolvimento cognitivo dos alunos das instituições particulares é intensificada pela massificação e mercantilização do Ensino Superior por elas oferecido. Pode-se dizer que tais fatores contribuem para o distanciamento da concepção de qualidade, para o insucesso da formação profissional e para a falta de autonomia dos discentes, percebida como o fator crítico de sucesso pelo mercado de trabalho.

Alguns acadêmicos, principalmente os do primeiro ano dos cursos de graduação, apresentam determinadas dificuldades relacionadas à adaptação ao ambiente acadêmico, seja por questões relativas aos conhecimentos construídos na Educação Básica, seja por situação econômica ou por outros motivos que possam ter relação com o cognitivo, o social e até mesmo com o emocional e afetivo.

Atualmente é cada vez mais crescente a preocupação das Instituições de Ensino Superior com o processo de ensino e aprendizagem, diante das dificuldades constatadas em sala de aula. Considerando que o Ensino Superior responde pela formação de profissionais cada vez mais especializados, flexíveis e adaptáveis à nova configuração social e que as instituições devem desenvolver formas de atuação para o atendimento a esta nova realidade, Genghini, (2006) escreveu os fatores que interferem no rendimento escolar. Segundo a autora, as dificuldades de aprendizagem acontecem em diversos âmbitos, a saber:

No âmbito pessoal, os fatores que alteram o processo de produção acadêmica e/ ou a inexistência de um serviço de apoio ao estudante universitário deixam o aluno vulnerável às pressões decorrentes do Ensino Superior; No âmbito institucional, os sintomas manifestos de dificuldades de aprendizagem - baixo rendimento nas avaliações disciplinares, falta de motivação para acompanhar o ritmo das aulas e executar as atividades, pesquisas, laboratórios entre outras atividades próprias ao trabalho; No âmbito social, deixar o fenômeno crescer e se expandir, negando-lhe a existência em 
nossas instituições é o mesmo que ir à contramão da política para o Ensino Superior brasileiro, ante os desafios do novo século, que vinculam diretamente os resultados do desenvolvimento econômico e social de uma nação à qualidade e à expansão da educação e cultura. (GENGHINI, 2006, p. 23); No âmbito legal, o baixo rendimento escolar em alunos universitários dificulta e, em alguns casos até pode inviabilizar o cumprimento, por parte das Instituições de Ensino Superior.

Em sua tese de doutorado, Chaleta, (2002) apontou que os problemas de natureza acadêmica, tais como a falta de métodos de trabalho, estudo e de organização dos tempos e das tarefas atribuídas são responsáveis por uma boa parte dos níveis de insucesso registrados, o que foi corroborado pelas pesquisas de Almeida e Silva (2005) junto a alunos de curso superior. As principais queixas trazidas foram a colocação da falta de hábitos de estudo sistematizado, desorganização no método e com materiais acadêmicos, cobrança excessiva quanto ao próprio desempenho, dificuldade de acompanhar o ritmo da turma, sensação de isolamento, inadequação e desejo de abandonar a prática educativa. Alguns colocaram que "não sabiam estudar", pois nunca precisaram antes, sendo que a simples presença e atenção nas aulas eram suficientes para alcançar êxito nas provas e trabalhos. Outros indicaram desmotivação quanto aos conteúdos estudados, não conseguindo relacioná-los com aspectos do cotidiano da profissão futura. (CHALETA, 2002).

Outra questão que interfere no processo de aprendizagem acadêmica é a bagagem intelectual que os alunos trazem da Educação Básica. Uma pesquisa realizada no site da Faculdade de Direito Promove, Instituição de Ensino Superior particular, revelou que mais de $80 \%$ dos alunos são egressos da escola pública. Esses estudantes refletem em sala de aula o legado de uma educação pública que há muito deixa a desejar. Os reflexos mais perceptíveis são a falta de motivação e de bases intelectuais anteriores, bem como dificuldades na leitura, compreensão e escrita, que podem igualmente ter consequências graves no sucesso acadêmico. Com relação a esse fator, as Instituições de Ensino Superior podem amenizar os efeitos ao propor disciplinas extracurriculares de nivelamento, monitorias, grupos de estudos ou estratégias afins. Isso seria eficaz na medida em que houver o interesse por parte do aluno em reconhecer tais deficiências. Segundo Tavares et al (2008, p. 21), “[...] as principais reflexões e ações sobre a temática da aprendizagem no Ensino Superior apontam para estratégias multifacetadas e globais, envolvendo todos os atores deste processo: docentes, alunos, currículos, instituições e contextos de aprendizagem". 
Partindo dessas considerações iniciais, o trabalho ora apresentado, além de identificação na literatura nacional, estudos referentes às dificuldades da aprendizagem no contexto do ensino superior, a apresentação do conceito de psicoterapia breve e outras modalidades de atendimento associadas às dificuldades de aprendizagem apresentadas por acadêmicos, buscará articular vinhetas de dados coletados em serviços de atendimento psicológico a esses sujeitos.

\section{Método}

Trata-se de estudo de revisão de literatura sobre psicoterapia breve e dificuldade de aprendizagem em acadêmicos do nível superior associada a uma discussão de dados documentais oriunda de 02 (dois) serviços de atendimento psicológico a discentes e outros atores do contexto universitário, numa vertente predominantemente qualitativa.

\section{Local e participantes}

As instituições de base deste estudo são 02 (duas) faculdades privadas de ensino superior e não terão seus nomes identificados, no entanto, baseou-se em práticas de dois profissionais e seus respectivos relatórios de serviços.

\section{Procedimentos e materiais}

Para o levantamento do material a ser empregado na revisão da literatura acerca do emprego da psicoterapia breve em âmbito acadêmico foram feitas buscas de artigos científicos na Biblioteca Virtual da Saúde - BVS e Google acadêmico com os seguintes descritores: Psicoterapia Breve e Dificuldade de Aprendizagem em estudantes do Ensino Superior; Quanto ao aspecto documental, realizou-se coleta de dados a partir de 2 relatórios de atendimentos realizados por serviço de atendimento em Psicologia de duas faculdades privadas. 


\section{Resultados e discussões}

Na sequência, faremos uma discussão acerca da literatura científica consultada, para apontar elementos de atendimentos realizados nos serviços especializados, nas duas instituições de ensino superior.

\section{Elementos da literatura nacional}

Dos resultados obtidos com a palavra-chave "Dificuldade de Aprendizagem em Acadêmicos do Nível Superior" foram encontrados 20 artigos. Dos 20 (vinte), 7 (sete) foram excluídos devido a abordagem da dificuldade de aprendizagem na fase escolar, e 8 (oito ) por abordar assuntos diversos como por exemplo a motivação educacional voltada para professores, portanto, somente 5 artigos foram selecionados para discussão do trabalho proposto. Os artigos pesquisados com as palavras-chaves tanto Psicoterapia Breve quanto para a Dificuldade de Aprendizagem em acadêmicos do Ensino Superior obteve-se um resultado total de 80 artigos. Segue abaixo a Tabela 1 que traz os artigos encontrados com a palavra-chave "dificuldade de aprendizado no Ensino Superior”.

Tabela 1: Artigos encontrados com a palavra-chave "Dificuldade de aprendizado no Ensino Superior"

\begin{tabular}{|c|c|c|c|}
\hline & Autor/Ano & Método & Resultados \\
\hline 1 & BORINE (2013). & Revisão de Literatura. & $\begin{array}{l}\text {-As dificuldades são diversas e } \\
\text { acumulativas, podendo o mesmo indivíduo } \\
\text { apresentar uma ou mais variáveis de } \\
\text { dificuldade. } \\
\text { - Foi possível diferenciar dificuldade de } \\
\text { distúrbio de aprendizagem. }\end{array}$ \\
\hline 2 & CARELLI (1999). & Pesquisa de Campo & $\begin{array}{l}\text { - A razão principal da insuficiência de } \\
\text { tempo para os estudos, principalmente entre } \\
\text { os alunos do noturno, é atribuída ao fato de } \\
\text { terem que dedicar grande palie do seu } \\
\text { tempo ao trabalho. } \\
\text { - o sono e o cansaço foram os fatores } \\
\text { apontados como os mais comprometedores } \\
\text { do rendimento acadêmico pelos alunos do } \\
\text { noturno. }\end{array}$ \\
\hline 3 & CAPELLINI (2009). & Pesquisa de Campo & $\begin{array}{l}\text { - os escolares com dificuldades de } \\
\text { aprendizagem } \\
\text { comprometimento na relação entre as } \\
\text { capacidades de nomeação e automatização } \\
\text { dos estímulos apresentados com a } \\
\text { capacidade de acesso lexical, discriminação }\end{array}$ \\
\hline
\end{tabular}




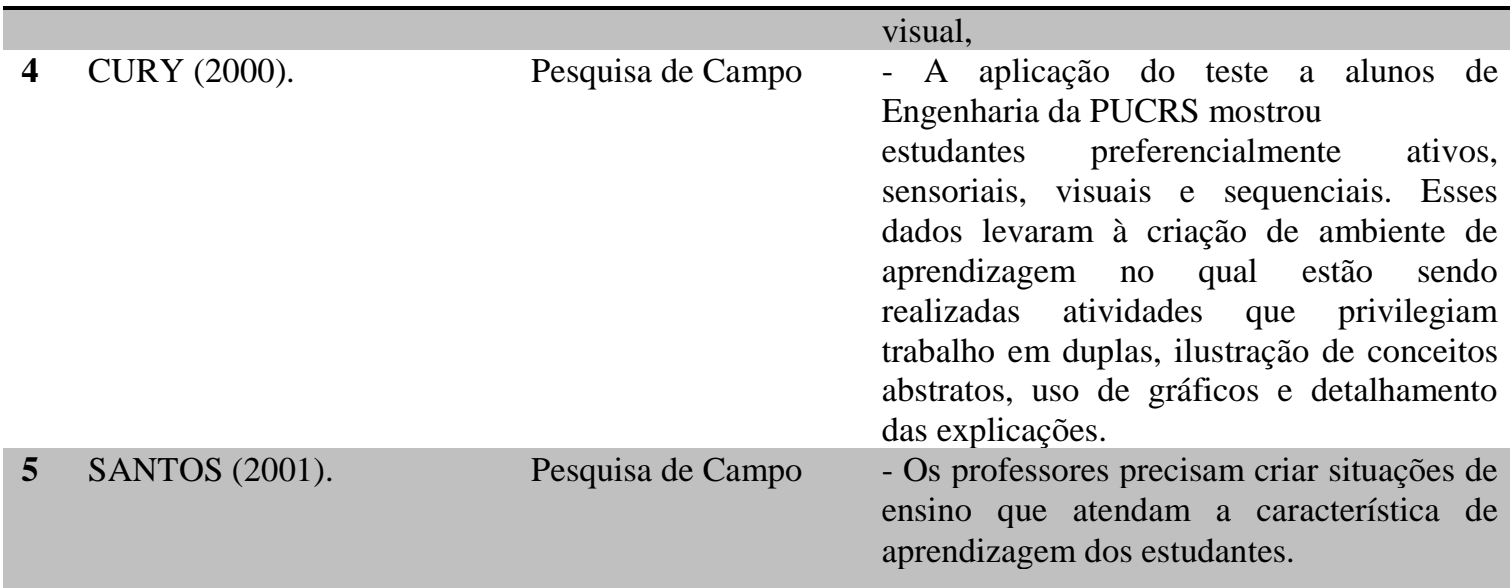

Fonte: Adaptado pelos autores

As dificuldades de aprendizagem podem ser entendidas como obstáculos ou barreiras encontradas por alunos durante o período de escolarização, referentes à captação ou à assimilação dos conteúdos propostos. Essas dificuldades podem ser duradouras ou passageiras e mais ou menos intensas (CAPELLINE, 2009).

\section{Caracterização de queixas discentes decorrentes de atendimentos psicológicos}

$\mathrm{Na}$ sequencia, abordaremos um conjunto de elementos formulados como "queixas" "de discentes em 2 (dois) serviços de atendimento psicológico de duas faculdades privadas, dentro do que pretendemos constituir como uma 'tipologia de queixas acadêmicas em serviços de psicologia':

Tabela 2: Elementos formulados - queixas acadêmicas

\begin{tabular}{ll}
\hline \multicolumn{1}{c}{ Categorias de Queixas } & \multicolumn{1}{c}{ Descrição } \\
\hline Aceitação /Autoestima & Questões que envolvem problemas intrapessoais \\
Relacionamento & Questões que envolvem problemas nas relações interpessoais sociais \\
interpessoal/autoestima & \\
Relacionamento amoroso & Questões que envolvem problemas nas relações afetivo-amorosas \\
Relacionamento familiar & Questões que envolvem problemas nas relações familiares \\
Depressão/estresse laboral & Questões que envolvem problemas nas relações de trabalho \\
Insegurança/ Ansiedade/ Estudos & Questões que envolvem problemas imediatamente ligadas à vivência \\
& acadêmica
\end{tabular}

3 Salientamos que ainda não dispomos de uma sistematização quantitativa desses dados, mas que se buscará na sequência dos atendimentos do serviço. 
Disgrafia/Disortografia/Dislexia

Questões que envolvem problemas de constituição psicobiológicas

Saúde (Crises de enxaqueca, Questões que envolvem problemas emergenciais de saúde

náuseas)

Fonte: elaborado pelos autores

Importante destacar que as categorias listadas acima possuem um caráter didático e, portanto, sem rigidez de separação entre si, posto que estão em permanente interação. Assim, uma queixa inicial de dificuldade de aprendizagem de um conteúdo pode, a partir da própria elaboração do sujeito, apontar para questões ansiógenas anteriores e relacionadas às suas relações inter-intrapessoais ou de modificações no seu desenvolvimento geral por exemplo.

Deste modo, uma possibilidade de tipologia das queixas seria o agrupamento em

1 - Questões interpessoais: 1.1 Acadêmicos: (Problemas de relacionamentos com colegas de sala, grupos de trabalho, amorosos, com professores etc); 1.2 - Familiares: Problemas de relacionamentos com filhos, pais e outros familiares; 1.3 - Amorosos: Problemas de relacionamentos com pares amorosos, cônjuges etc. 2 - Questões intrapessoais: 2.1 - Mudanças em fases de desenvolvimento, de identidade, autonomia x heteronomia, questões afetivas; 2.2 Questões cognitivas-acadêmicas diretas: dificuldade cognitiva de compreensão de conteúdos ministrados, em relação à escolha de curso, exigências do curso e perspectiva profissional; 3 - Questões sociais: Dificuldade em conseguir trabalho e manter pagamento do curso etc; 4 - Questões de doença: Problemas imediatos de saúde física.

\section{Sobre os atendimentos discentes}

Para cada caso atendido, são realizados um acolhimento, escuta da queixa inicial e breve anamnese; ao final da primeira sessão, cada acadêmico poderá, por si mesmo, sinalizar a necessidade de continuar os próximos atendimentos limitados a 04 sessões.

Os atendimentos, embora não sejam uma psicoterapia, são psicoterapêuticos e podem ter efeitos imediatos sobre as queixas/problemas formulados pelos discentes, ajudando-os a organizar os diversos aspectos relacionais, emocionais e acadêmicos, com novas formulações e posicionamentos perante a realidade. Destacamos que o serviço pode ainda ajudar ao acadêmico na formulação de uma demanda para prosseguimento 
de psicoterapia em outro local, inclusive para a Clínica de Psicologia das faculdades. Deste modo, os atendimentos em sua configuração breve e em poucas sessões tiveram o efeito de escoar o tensionamento dos momentos ansiógenos e de crise, favorecendo insights e alívio.

\section{Os atendimentos para-além dos discentes}

Embora, esses serviços girem em torno do discente como elemento-central no processo de ensino-aprendizagem, houveram atendimentos a outros atores envolvidos neste processo, sejam coordenadores de curso, coordenação pedagógica, docentes, pais , familiares e funcionários . E, além dos atendimentos individuais, esses serviços demandam outras modalidades de intervenção, como por exemplo, a intervenção grupal, a aplicação individualização de avaliação a discentes e a ministração de minicursos a discentes sobre desenvolvimento da aprendizagem.

\section{Considerações finais}

Pela pesquisa realizada nas duas IES e as teorias expostas neste artigo, podemos concluir que a inserção de uma visão psicológica, acompanhada de práticas pedagógicas no ensino superior, é uma necessidade para a diminuição das tensões e problemas que acontecem nesse âmbito de ensino.

Com os exemplos citados, vemos que a atuação psicológica nas duas vertentes do processo de ensino/aprendizagem: docentes e alunos, pode se tornar realidade, dependendo da gestão educacional empreendida pela instituição. Se ela considera que a aprendizagem deve ser significativa para o aprendiz mesmo no Ensino Superior, vai possibilitar a troca de experiências, a coerência com as expectativas do mercado de trabalho, a disponibilidade para resolver problemas e aprender novos assuntos, a articulação entre os conhecimentos que estão sendo construídos e aqueles que já foram adquiridos.

As Instituições de Ensino Superior que agem desta maneira pressupõem ainda que as teorias vão ganhando sentido quando são alocadas em contextos reais e nas 
interações com as práticas sociais e podem se transformar em práxis educativas inovadoras e renovadoras de esperanças e sucessos pessoais e profissionais.

Observa-se também que a dificuldade de aprendizagem dos acadêmicos pode estar diretamente relacionada com fatores intrínsecos, ou seja, emocionais, que o afetam e o bloqueiam de alguma forma, sendo estratégico e de grande relevância a introdução de apoio psicológico e acompanhamento através de psicoterapias com profissionais previamente qualificados.

\section{REFERÊNCIAS}

ALMEIDA, F. de.; SILVA, M. B. Psicopedagogia para adultos - psicoandragogia: uma proposta de atendimento psicopedagógico para adolescentes e adultos jovens. RUBS, Curitiba, v.1, n.4, sup.1, p.46-48, out./dez. 2005

BORINE, R. C. C.; Considerações gerais sobre dificuldade de aprendizagem: conceitos e fatores de risco. Escola Superior Aberta do Brasil- ESAB; Curso de pósgraduação Lato Sensu em psicopedagogia clínica e institucional. Vila Velha (ES), 2013.

BORTOLANZA, M. L. Insucesso acadêmico na universidade abordagens psicopedagógicas. Erechim/RS: Edifapes, 2002.

BOSSA, N. A. Dificuldades de aprendizagem: O que são? Como tratá-las? Porto Alegre: Artes Médicas, 2000.

BRAIER, E. A. Psicoterapia breve de orientação psicanalítica. São Paulo: Martins Fontes, 2008.

BRASIL. LEI no 9.394, de 20 de dezembro de 1996. D.O.U., de 23 de dezembro de 1996.

CAPELlini, S. A.; CONRADO, T. L. B. C. Desempenho de escolares com e sem dificuldades de aprendizagem de ensino particular em habilidade fonológica, nomeação rápida,leitura e escrita. Rev CEFAC, v.11, Supl2, p. 183-193, 2009.

CARElli, M, J, G. ACÁCiA, A, A, S. Condições temporais e pessoais de estudos em universitários. Universidade São Francisco, 1999.

CARVALHO, D. B. de.; SANTANA, J. M.; SANTANA, V. M. de. Humanização e controle social: o psicólogo como ouvidor hospitalar. Psicologia Ciência e Profissão, vol. 29 (1), 172-183, 2009

CASTRO, E. K. de.; BORNHOLDT, E. Psicologia da saúde x Psicologia hospitalar: definições e possibilidades de inserção profissional. Revista Psicologia: Ciência e Profissão. V. 24. N. 3. Brasília: setembro, 2004. 
CHALETA, M. E. R. Abordagens ao estudo e estratégias de aprendizagem no ensino superior. Évora: Universidade de Évora - Tese de Doutorado, 2002.

CIASCA, S. M. Diagnóstico dos distúrbios de aprendizagem em crianças: análise de uma prática interdisciplinar. Dissertação (Mestrado em Psicologia) -Universidade de São Paulo, 1999.

CURY, H. N. Estilos de aprendizagem de alunos de engenharia. Anais XXVIII Congresso Brasileiro de Ensino de Engenharia. Ouro Preto, 2000.

FERRAZ, M.; PEREIRA, A. A dinâmica da personalidade e o homesikness (saudades de casa) dos jovens estudantes universitários. Revista Psicologia Saúde e Doenças, p. 149 a 164. 2002.

FIORINI, H. J. Teoria e técnica de psicoterapias. São Paulo: Martins Fontes, 2008.

FONSECA, V. Introdução às dificuldades de aprendizagem. Porto Alegre: Artes Médicas, 2009.

GENGHINI, E. B. O Ensino Superior no Brasil: Fatores que interferem no rendimento escolar e a visão dos alunos sobre suas dificuldades de aprendizagem. Revista da Educação: Universidade de Guarulhos, p. 18- 30. 2006.

HEGENBERG, M. Psicoterapia breve: clínica psicanalítica. 3 ed. Casa do Psicólogo 2010.

HILGARD, E. F. Teorias da aprendizagem. EPU, São Paulo, 1973.

JOSÉ, E. A.; COELHO, M. T. Problemas de aprendizagem. São Paulo, Ática. 1989.

KNOBEL, M. Psicoterapia breve. Vol 14. Coleção Temas Básicos de Psicologia. 2 ed. São Paulo: EPU, 1986.

MALAN, D.; SELVA, P. C. D. Psicoterapia dinâmica intensiva breve: um método inovador. Porto Alegre, RS: ArtMed, 2008.

PAPALIA, D; OLDS, S. W. Desenvolvimento humano. 7. ed. Porto Alegre: Artmed, 2000.

RIBEIRO, V. M. (Org.). Letramento no Brasil. São Paulo: Global, 2003.

SIMON, Ryad. Psicoterapia breve operacionalizada: teoria e técnica. São Paulo: Casa do Psicólogo, 2011.

TAVARES, J. et al. Docência e Aprendizagem no Ensino Superior. In: Investigar em educação. Revista da Sociedade Portuguesa de Ciências da Educação, n. ${ }^{\circ}$ 3, Junho. Lisboa: SPCE, 2008 p. 15 -55.

YOSHIDA, E. M. P. Significância clínica de mudança em processo de psicoterapia psicodinâmica breve. Paideia, São Paulo, vol. 18 (40), p. 305-316. 2008. 
ZANOLlA, Silvia Rosa da Silva. O conceito de mediação em Vigotski e Adorno. Psicol. Soc., Belo Horizonte, v. 24, n. 1, abr. 2012. Disponível em: $<$ http://www.scielo.br/scielo. $\quad$ php?script=sci_arttext\&pid=S0102$71822012000100002 \& \operatorname{lng}=e n \& n r m=i s o>$. Acesso em: 1 jun. 2014.

\section{Como referenciar este artigo}

LIZARDO DE ASSIS, Cleber; AYRES, Núbia Roberta. Atendimento Psicológico e Demandas Discentes a partir de serviços em Faculdades Privadas do norte do país. Revista on line de Política e Gestão Educacional, Araraquara, v.21, n.2, p. 383-397, 2017. Disponível em: <http://dx.doi.org/10.22633/rpge.v21.n.2.2017.10012>. ISSN: 1519-9029.

Submetido em: 27/05/2017

Aprovado em: 27/07/2017 